\title{
Transcatheter versus surgical aortic valve replacement in intermediate risk patients: a meta-analysis
}

\author{
Sameer Arora ${ }^{1,2}$, Jacob A. Misenheimer ${ }^{3}$, Wesley Jones ${ }^{1}$, Amol Bahekar ${ }^{2}$, Melissa Caughey ${ }^{3}$, Cassandra J. \\ Ramm $^{3}$, Thomas G. Caranasos ${ }^{3}$, Michael Yeung ${ }^{3}$, John P. Vavalle ${ }^{3}$ \\ ${ }^{1}$ Campbell University School of Osteopathic Medicine, Lillington, NC, USA; ${ }^{2}$ Cape Fear Valley Medical Center, Fayetteville, NC, USA; ${ }^{3}$ University \\ of North Carolina, Chapel Hill, NC, USA \\ Contributions: (I) Conception and design: JP Vavalle, S Arora; (II) Administrative support: None; (III) Provision of study materials or patients: JP \\ Vavalle; (IV) Collection and assembly of data: S Arora, JP Vavalle; (V) Data analysis and interpretation: S Arora, JA Misenheimer, W Jones, A \\ Bahekar, M Caughey, CJ Ramm, TG Caranasos, M Yeung; (VI) Manuscript writing: All authors; (VII) Final approval of manuscript: All authors. \\ Correspondence to: Sameer Arora, MD. 212 Aldenwood Place, Apex, NC 27539, USA. Email: sameerarora.cj@gmail.com.
}

Background: Transcatheter aortic valve replacement (TAVR) has been approved in patients with high or prohibited surgical risk for surgery for treatment of severe symptomatic aortic stenosis. Prospective studies examining the benefits of TAVR in intermediate risk patients are ongoing. Other smaller studies including lower risk patients have been conducted, but further meta-analysis of these studies is required to draw more broad comparisons.

Methods: A Medline search was conducted using standard methodology to search for clinical trials and observational studies including intermediate risk patients. We limited our meta-analysis to studies matching patient populations by propensity scores or randomization and examined clinical outcomes between TAVR and surgical aortic valve replacement (SAVR).

Results: Analysis of the TAVR and SAVR cohorts revealed no significant differences in the outcomes of 30-day [OR (95\% CI): $0.85(0.57,1.26)$ ] or 1-year mortality [OR $(95 \% \mathrm{CI}): 0.96(0.75,1.23)]$. A trend towards benefit with TAVR was noted in terms of neurological events and myocardial infarction (MI) without statistical significance. A statistically significant decrease in risk of post-procedural acute renal failure in the TAVR group [OR (95\% CI): $0.52(0.27,0.99)$ ] was observed, but so was a significantly higher rate of pacemaker implantations for the TAVR group [OR (95\% CI): $6.51(3.23,13.12)]$.

Conclusions: We conclude that in intermediate risk patients undergoing aortic valve replacement, the risk of mortality, neurological outcomes, and MI do not appear to be significantly different between TAVR and SAVR. However, there appears to be a significant reduction in risk of acute renal failure at the expense of an increased risk of requiring a permanent pacemaker in low and intermediate risk patients undergoing TAVR compared to SAVR.

Keywords: Transcatheter aortic valve replacement (TAVR); aortic stenosis; surgical risk; surgical aortic valve replacement (SAVR)

Submitted Feb 19, 2016. Accepted for publication Mar 03, 2016.

doi: $10.21037 / \mathrm{cdt} .2016 .03 .04$

View this article at: http://dx.doi.org/10.21037/cdt.2016.03.04 


\section{Introduction}

TAVR was first described in 2002 by Alain Cribier and colleagues in a 57 -year-old man with a severely calcified, bicuspid aortic valve (1). Although their transvenous approach was met with limitations, Webb et al. later reported improved safety of TAVR via a transfemoral arterial approach (2). The advent of TAVR raised the hope for an alternative, less invasive treatment for aortic stenosis. Since then, the success of TAVR has been consolidated with the help of clinical trials. The Placement of Aortic Transcatheter Valve Trial (PARTNER) revealed TAVR to be non-inferior to surgical aortic valve replacement (SAVR) and superior to medical therapy for high risk and prohibitive risk surgical patients who suffered from severe, symptomatic aortic stenosis, respectively (3). The success of TAVR was further proven by the CoreValve US Pivotal Study, which indicated superiority of TAVR over SAVR in regards to mortality in patients with increased risk of death from surgery (4). This has led to the recognition of TAVR as the treatment of choice for aortic valve replacement for patients with severe, symptomatic aortic stenosis who are considered high or prohibitive risk for SAVR (5). Although SAVR currently remains the standard of care for the intermediate to low surgical risk population, several independent studies conducted predominantly in Europe have compared TAVR to SAVR in this population and demonstrated promising results for TAVR. The influx of data on outcomes from TAVR in the intermediate risk population continues to increase (6-14). For example, the ongoing SURTAVI and PARTNER II trials are investigating the safety and efficacy of TAVR compared to SAVR in an intermediate risk population. Although a number of small trials comparing outcomes between TAVR and SAVR in this population exist, only a single, small metaanalysis of these studies has been performed to date (15). A larger, more comprehensive meta-analysis is necessary to focus specifically on the population considered intermediate risk for valve replacement surgery.

\section{Methods}

\section{Search strategy}

We searched Medline, EMBASE, Google Scholar, Web of Science and Cochrane databases for studies with key words transcatheter, transact heter aortic valve implantation, percutaneous aortic valve implantation, percutaneous aortic valve replacement, transcatheter aortic valve replacement
(TAVR), TAVI, TAVR, low, intermediate, moderate and propensity. All retrieved abstracts were reviewed by two authors independently and later again confirmed by a third author. The bibliographies of all articles were reviewed to look for other potential articles with eligible data for the meta-analysis. The meta-analysis has been reported according to Meta-analysis of observational studies in Epidemiology guidelines.

\section{Study characteristics}

All randomized control trials (RCTs) and studies using propensity score matching based on clinical characteristics and surgical risk scores, i.e., the Society of Thoracic Surgeons (STS) Score or Euroscore, and categorizing patients into intermediate risk groups were included in the study. We gave priority to the STS score if both scores were available as it has been demonstrated to be superior to Euroscore in predicting outcomes $(16,17)$. STS predicted risk of mortality (PROM) (mean $<8 \%)$ was used as a benchmark for inclusion on the basis of the SURTAVI definition of the intermediate risk categories. Euroscore (mean $<20 \%$ ) was used only if the STS score was not available. Inclusion was restricted to comparison studies only and studies with isolated data from only one of the two interventions were not included $(13,14,18,19)$. We included all studies irrespective of the type of TAVR valve and the route of vascular access. The study characteristics and the distribution of patients between the two study groups are demonstrated in Table 1.

\section{Outcome measures}

The Valve Academic Research Consortium (VARC) in its most recent update has defined clinical endpoints to help future clinical trials standardize outcomes when using TAVR as intervention (20). Even though some of our studies were conducted before the most recent updates from VARC, they still primarily used VARC endpoints to quantify outcomes. We pooled data from individual studies to determine 30-day mortality, 1-year mortality, stroke and all neurological events at 30 days, $\mathrm{MI}$ at 30 days, acute renal failure at 30 days, and pacemaker implantation at 30 days.

\section{Meta-analysis}

Random effects meta-analyses were carried out using RevMan 5.2 (Cochrane, Oxford, UK). The presence and 


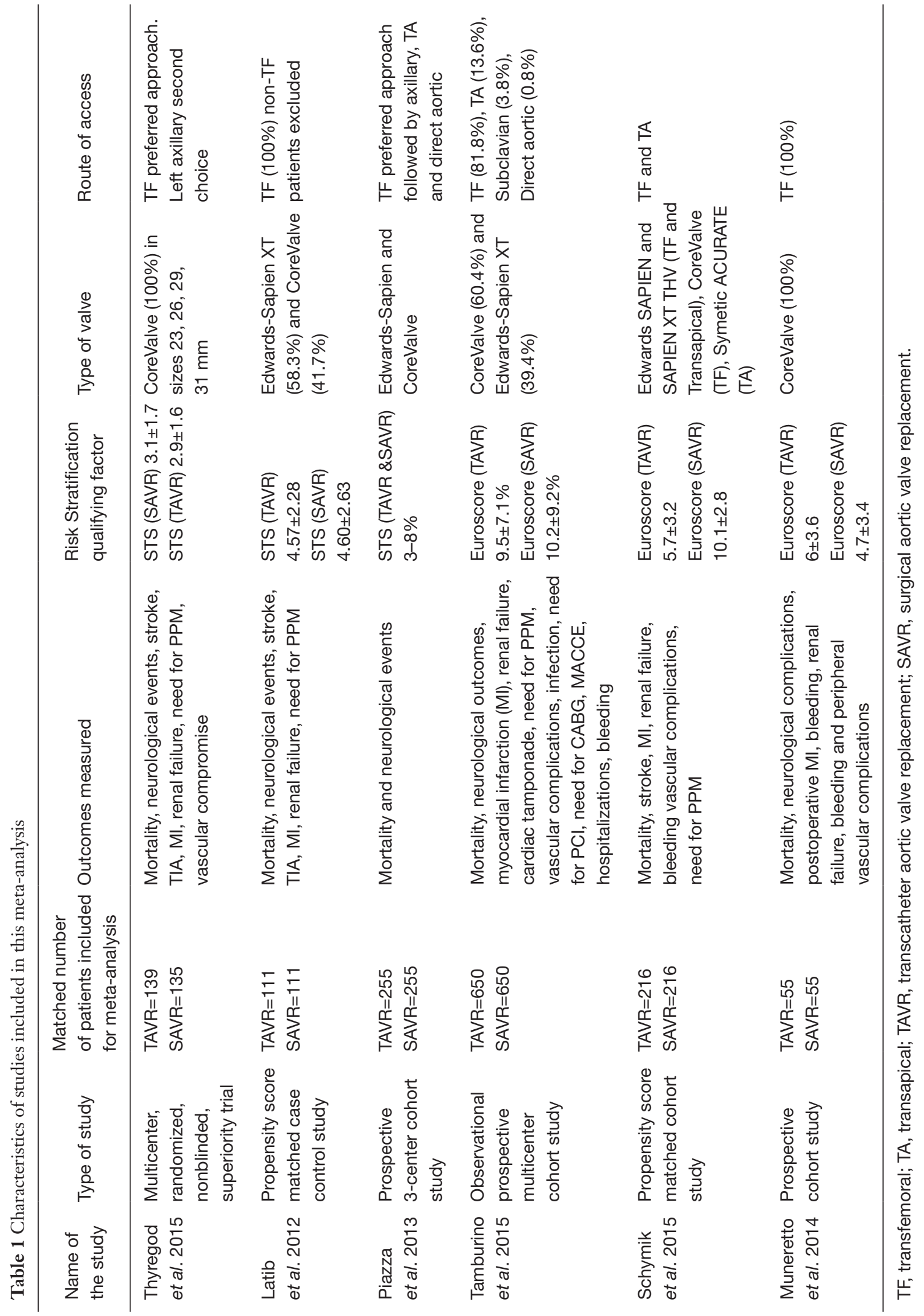




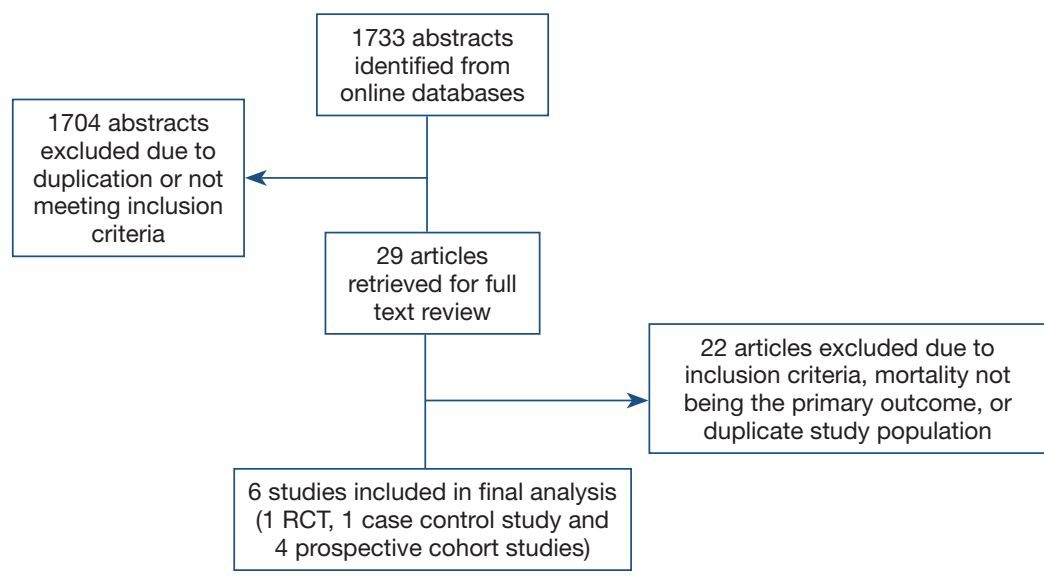

Figure 1 Flow diagram of selected studies. Displayed is the methodology of screening and selecting appropriate studies meeting the inclusion criteria to be included in the final meta-analysis.

degree of between-study heterogeneity was assessed using Cochran's $Q$ statistic $(\mathrm{P}<0.10)$ and $\mathrm{I}^{2}$ values. Due to inherent differences in study populations and clinical sites, random effects meta-analyses were conducted for all outcomes. Odds ratios of 30-day mortality, 1-year mortality, and 30-day adverse events were meta-analyzed, with summaries representing the mean of the random effects distributions. Because the majority of studies reported adverse events by the number of occurrences, 30-day odds ratios were analyzed without consideration of competing risks. Publication bias was assessed using Harbord's modified test for small-study effects, with 30-day mortality considered the primary outcome.

\section{Results}

\section{Study population}

A total of 1,733 abstracts were identified with a literature search, of which 29 full articles were retrieved and reviewed in depth. A total of 6 publications (1 RCT and 5 propensity score matched observational studies) were identified for inclusion (Figure 1) (6-8,10-12). Outcomes were abstracted and meta-analyzed if reported by a minimum of 4 studies. Consistent with the current Food and Drug Administration (FDA) restrictions for TAVR in low to intermediate risk patients in the United States, all studies originated from Europe ( 3 in Italy, 2 in Germany, and 1 in Denmark). A total of 2,848 patients were propensity score matched in the included studies and were included in the meta-analysis. Duplicate data was not accepted. There was no evidence of publication bias detected $(\mathrm{P}=0.8)$. Study population demographics were similar, with average ages ranging from $78-81$ years and percentage of women ranging from $47 \%$ to $59 \%$. Of the studies reporting baseline comorbidities, diabetes prevalence ranged from $20 \%$ to $25 \%$, COPD prevalence was $12 \%$ to $37 \%$, prevalence of peripheral vascular disease was $5 \%$ to $30 \%$, and the prevalence of prior myocardial infarction (MI) ranged from $5 \%$ to $14 \%$ (Table 2). Full patient demographics and characteristics are listed in Table 2.

\section{Outcomes}

All studies reported 30-day mortality; however, 1-year mortality was limited to 4 publications. The overall 30-day mortality ranged from $0.9-7.5 \%$, increasing to $6.1-17.0 \%$ after a year. Mortality odds ratios comparing TAVR to SAVR were close to null (1.0) for all abstracted 30-day and 1 -year mortality outcomes (Figures 2,3 ). Thirty day adverse events were reported by 5 studies. Because the 30-day mortality rate among these studies was low (0.9-3.4\%), competing risk bias was considered minimal and outcomes were analyzed using odds ratios.

No significant difference was detected between TAVR $v$ s. SAVR at 30 days in regards to MI [OR (95\% CI): 0.48 (0.21, $1.11)]$, stroke [OR (95\% CI): $0.61(0.31,1.20)]$, or adverse neurological events [OR (95\% CI): $0.63(0.35,1.14)]$, but a trend towards fewer events in the TAVR group was observed with a $40-50 \%$ lower odds of developing these complications (Figures 4-6). There was also a 40-50\% lower odds of acute renal failure in the TAVR group as compared to the SAVR group at 30 days, which reached statistical 
Table 2 Baseline characteristics of 6 matched studies comparing transcatheter aortic valve replacement (TAVR) to surgical aortic valve replacement (SAVR)

\begin{tabular}{|c|c|c|c|}
\hline Characteristic & TAVR $(\mathrm{N}=1,426)$ average (range) & SAVR $(\mathrm{N}=1,422)$ average (range) & Number of studies \\
\hline Mean age (years) & 80 [78-81] & 79 [79-80] & $(6-8,10-12)$ \\
\hline Female (\%) & $54 \%(46-59 \%)$ & $53 \%(47-60 \%)$ & $(6-8,10-12)$ \\
\hline Coronary artery disease (\%) & $44 \%(30-57 \%)$ & $42 \%(15-58 \%)$ & $(6,7,11,12)$ \\
\hline Hypertension (\%) & $73 \%(66-86 \%)$ & $77 \%(69-82 \%)$ & $(6-8,12)$ \\
\hline Previous myocardial infarction (MI) (\%) & $7 \%(2-14 \%)$ & $9 \%(3-14 \%)$ & $(6,8,10-12)$ \\
\hline Previous Stroke/TIA (\%) & $10 \%(3-17 \%)$ & $10 \%(4-18 \%)$ & $(6-8,10,11)$ \\
\hline Chronic lung disease (\%) & $22 \%(9-47 \%)$ & $18 \%(9-27 \%)$ & $(6-8,10-12)$ \\
\hline Peripheral arteriopathy (\%) & $14 \%(4-26 \%)$ & $17 \%(7-34 \%)$ & $(6-8,10-12)$ \\
\hline NYHA III-IV (\%) & $61 \%(48-75 \%)$ & $64 \%(45-75 \%)$ & $(6-8,10,12)$ \\
\hline
\end{tabular}

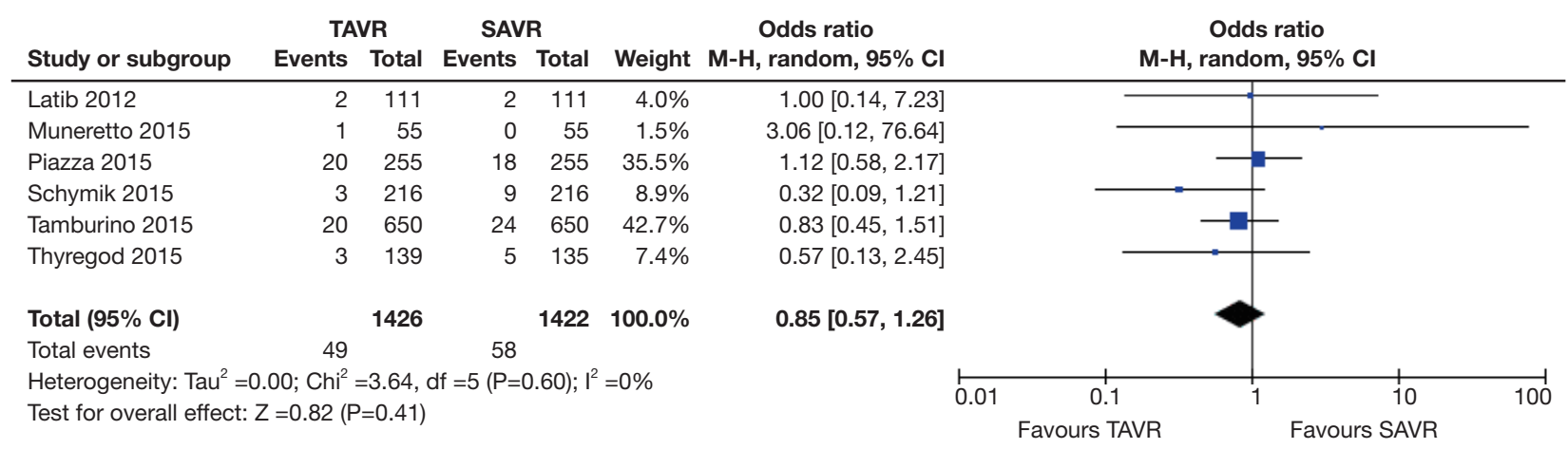

Figure 2 Thirty day mortality. Displayed is 30 days mortality of TAVR vs. SAVR in this intermediate risk population. TAVR, transcatheter aortic valve replacement; SAVR, surgical aortic valve replacement.

\begin{tabular}{|c|c|c|c|c|c|c|c|c|c|c|}
\hline \multirow[b]{2}{*}{ Study or subgroup } & \multicolumn{2}{|c|}{ TAVR } & \multicolumn{2}{|c|}{ SAVR } & \multicolumn{3}{|c|}{ Odds ratio } & \multirow{2}{*}{\multicolumn{2}{|c|}{$\begin{array}{c}\text { Odds ratio } \\
\mathrm{M}-\mathrm{H} \text {, random, } 95 \% \mathrm{Cl}\end{array}$}} & \\
\hline & Events & Total & Events & Total & Weight & $\mathrm{M}-\mathrm{H}$, random, $95 \% \mathrm{Cl}$ & & & & \\
\hline Thyregod 2015 & 7 & 139 & 10 & 135 & $6.3 \%$ & $0.66[0.24,1.80]$ & & & & \\
\hline Latib 2012 & 7 & 111 & 9 & 111 & $6.0 \%$ & $0.76[0.27,2.13]$ & & & & \\
\hline Piazza 2015 & 42 & 255 & 43 & 255 & $28.9 \%$ & $0.97[0.61,1.55]$ & & & & \\
\hline Tamburino 2015 & 83 & 650 & 82 & 650 & $58.8 \%$ & $1.01[0.73,1.41]$ & & & & \\
\hline Total $(95 \% \mathrm{Cl})$ & & 1155 & & 1151 & $100.0 \%$ & $0.96[0.75,1.23]$ & & & & \\
\hline Total events & 139 & & 144 & & & & & & & \\
\hline Heterogeneity: $\mathrm{Tau}^{2}=$ & $00 ; \mathrm{Chi}^{2}=$ & $=0.83, d$ & $\mathrm{df}=3(\mathrm{P}=\mathrm{C}$ & $.84) ; 1^{2}$ & $=0 \%$ & & & & & \\
\hline Test for overall effect & $=0.33(P$ & $=0.74)$ & & & & & 0.01 & 0.1 & 10 & 100 \\
\hline
\end{tabular}

Figure 3 One year mortality. Displayed is one year mortality of TAVR vs. SAVR in this intermediate risk population. TAVR, transcatheter aortic valve replacement; SAVR, surgical aortic valve replacement.

significance [OR (95\% CI): $0.51(0.27,0.99)]$ (Figure 7). Despite the apparent clinical benefit of TAVR over SAVR in these abstractions, TAVR was associated with an increased need for pacemaker implant within 30 days [OR (95\% CI): $6.51(3.23,13.12)]$ (Figure 8).

\section{Discussion}

The SURTAVI trial is an ongoing multicenter trial comparing the clinical outcomes for transcatheter and surgical valve replacement in intermediate risk patients defined by a STS risk score of $3-8 \%$. As we await the 


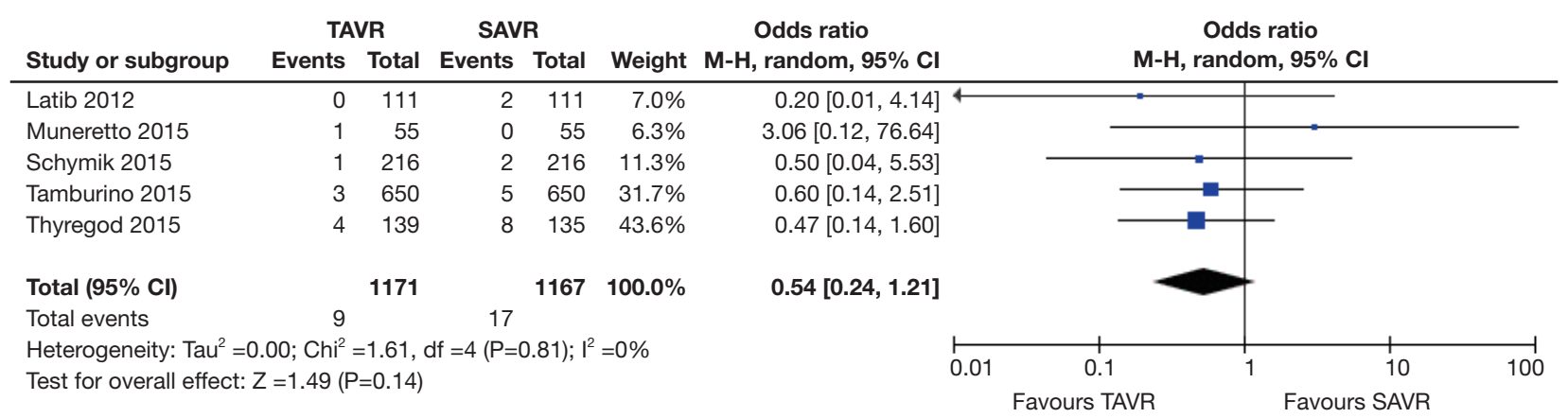

Figure 4 Myocardial Infarction (MI) at 30 days. Displayed is MI at 30 days of TAVR $v s$. SAVR in this intermediate risk population. TAVR, transcatheter aortic valve replacement; SAVR, surgical aortic valve replacement.

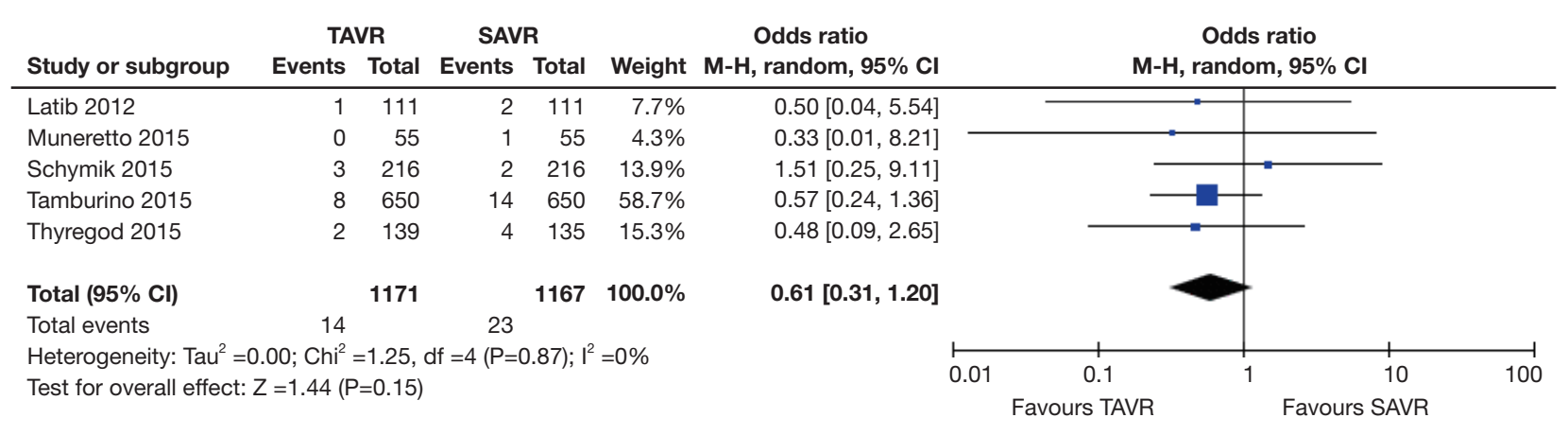

Figure 5 Stroke at 30 days. Displayed is stroke at 30 days of TAVR vs. SAVR in this intermediate risk population. TAVR, transcatheter aortic valve replacement; SAVR, surgical aortic valve replacement.

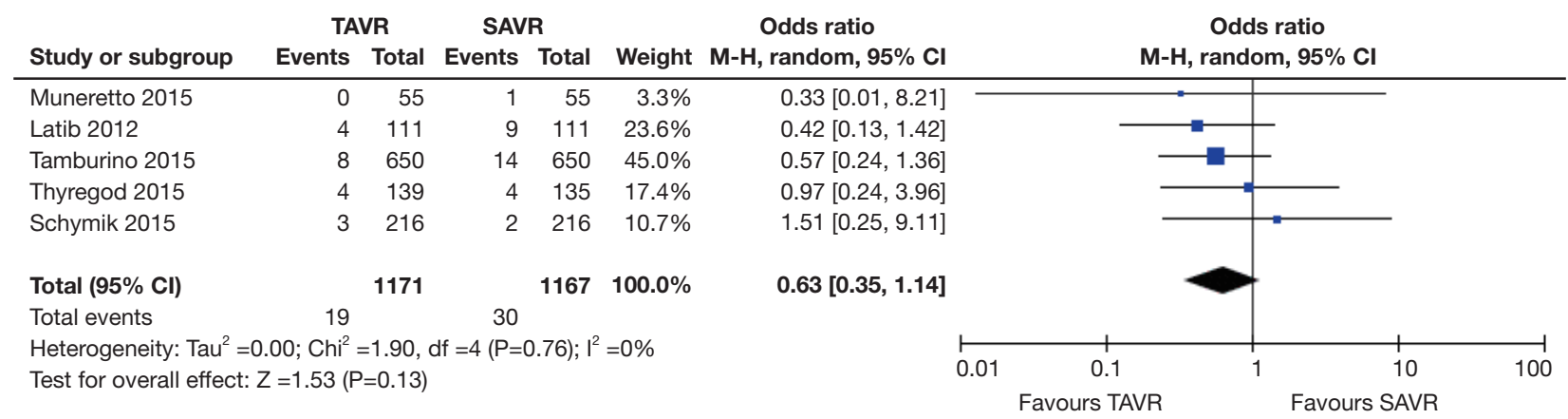

Figure 6 Neurological Events at 30 days. Displayed are neurological events at 30 days of TAVR vs. SAVR in this intermediate risk population. TAVR, transcatheter aortic valve replacement; SAVR, surgical aortic valve replacement.

results of this study, we designed a meta-analysis of trials comparing the two interventions in intermediate surgical risk patients in order to draw broader conclusions. This study design differed from that of the only previous, smaller meta-analysis in that we took into consideration the standard deviation for the risk scores (15). This was the reason for CoreValve US Pivotal Study not meeting the inclusion criteria (4). The results of the STACCATO trial were also not included in our meta-analysis as the early termination of the study was felt to have a high likelihood of skewing the results. Our meta-analysis is also broader, including several studies that were not randomized, controlled clinical trials but that still yielded comparable data on lower risk patients (11-12). This resulted in a larger 


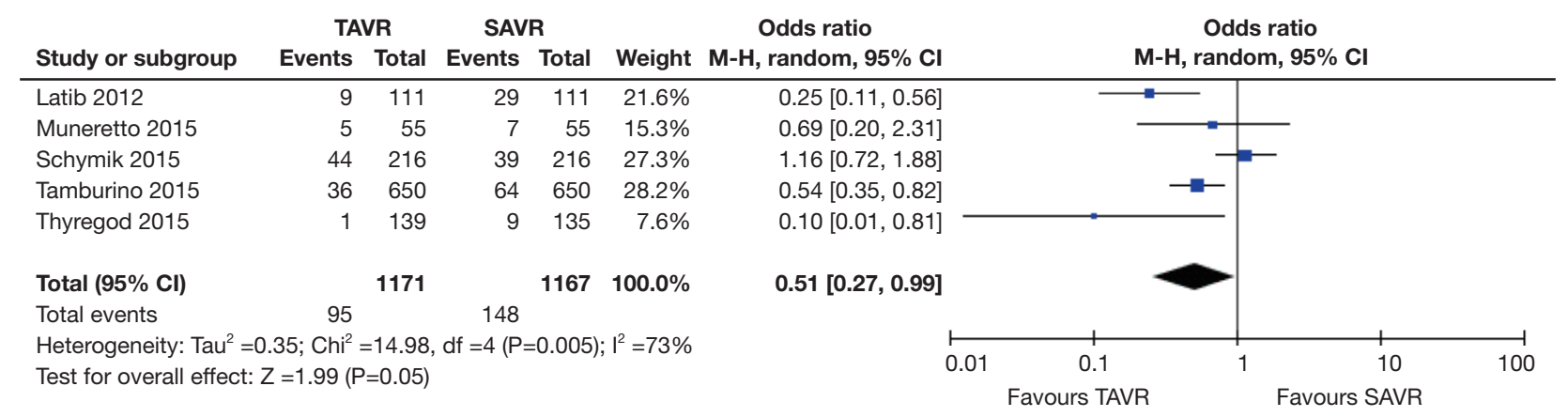

Figure 7 Acute Renal Failure at 30 days. Displayed is acute renal failure at 30 days of TAVR vs. SAVR in this intermediate risk population. TAVR, transcatheter aortic valve replacement; SAVR, surgical aortic valve replacement.

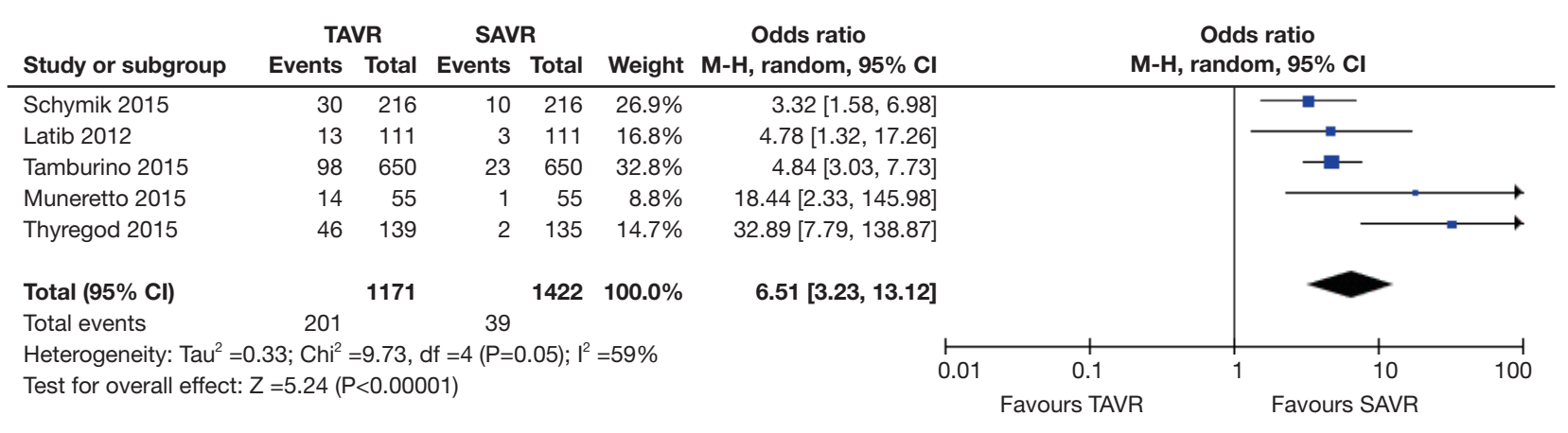

Figure 8 Pacemaker implantation at 30 days. Displayed is pacemaker implantation at 30 days of TAVR vs. SAVR in this intermediate risk population. TAVR, transcatheter aortic valve replacement; SAVR, surgical aortic valve replacement.

patient population and subsequently a meta-analysis with greater statistical power.

Although mortality, neurological outcomes, and MI were not significantly different between interventions, we did notice a trend favoring TAVR for 30-day MI and neurological outcomes. We did, however, find a significant decrease in acute renal failure and an increase in rates of pacemaker implantations in the TAVR group as compared to SAVR group. Although the incidence of acute renal failure has been examined in other trials (21-24), this is the first instance where a large data set has detected a significant benefit of TAVR over SAVR in a non-high risk population. Our data suggests that TAVR may result in improved neurologic outcomes and reduced post-procedure MIs but this study lacked the power to demonstrate a statistically significant difference. We await the results of ongoing randomized clinical trials to determine if these findings are supported.

Many of the serious complications of TAVR can be attributed to access site complications, bleeding, and poor positioning of the TAVR valve (15). This is an important consideration in regards to this data set, since it included outcomes of early first- and second-generation valves. Newer valve designs that allow smaller diameter access and the ability to fully recapture and reposition the valve are becoming standard of care, so operators can likely expect lower rates of related complications in the future. This is likely to lower the overall mortality and complication rates of the procedure in the future. Despite the fact that this data included older first- and second-generation valves, there was still a trend towards lower MI rates and neurological event rates. It is likely that as novel trials include newer valve designs that these outcomes favoring TAVR can be expected to improve significantly.

Although the PARTNER A trial failed to show a statistical difference in the incidence of pacemaker implantation between TAVR or SAVR (25), our analysis demonstrates an overwhelming increase in the rates of pacemaker implantations post-TAVR across the different studies [OR (95\% CI): 6.51 (3.23, 13.12)]. Another recent meta-analysis comparing self-expanding valves to balloonexpanding valves also demonstrated a higher incidence of rates of pacemaker implantation in patients with self- 
expending prosthesis, as has a small meta-analysis of all types of valves $(15,26)$. Significantly higher rates of pacemaker implantation were also noted in the CoreValve US Pivotal trial (4). Unlike the PARTNER A trial, our meta-analysis included patients receiving both balloonexpandable and self-expanding valves. Coupled with the results of the other meta-analyses and the CoreValve trial, the fact that the PARTNER A trial, which included exclusively balloon-expandable valves, did not reveal an increase pacemaker rate may indicate the risk is attributable to self-expanding valves. This inference must be placed in context, however, considering that newer generation balloon-expandable valves are also accepted to have a higher pacemaker implantation rate than SAVR (27).

Although it is a fairly comprehensive data set, this metaanalysis has several limitations. All of the trials included studied the use of earlier generation valves that are not currently the standard of practice. Although the newer valves may indeed have lower complication rates as compared to the first generation valves, this is not an absolute certainty, and definitive conclusions about the TAVR valves most commonly used today cannot be definitively drawn from this data. We assessed for publication bias, and although our results indicate publication bias is unlikely, it cannot be completely ruled out. Our meta-analysis also relied heavily on the Euroscore and STS score for risk stratification. Although these tools are the gold standard for surgical risk stratification for valve replacement, they have their own limitations. Specifically, they do not fully account for overall frailty and cognitive impairment, both of which contribute significantly to surgical risk. Subsequently, patients with low scores may have higher operative risk than the score itself may indicate, so our patient population could be somewhat higher risk than the scores would suggest. Despite these limitations, the data presented remain strong and yield more insight into the lower risk TAVR population, which has not been rigorously studied in the United States.

\section{Conclusions}

As we eagerly await the results of the SURTAVI and PARTNER II trials, we compared outcomes for patients who underwent TAVR as compared to SAVR in patients who are considered intermediate risk for surgery. Risk of mortality, neurological outcomes, and MI do not appear to be significantly different between the two groups, but there appears to be a significant reduction in risk of acute renal failure and an increased risk of requiring a permanent pacemaker in patients undergoing TAVR rather than SAVR.

\section{Acknowledgements}

None.

\section{Footnote}

Conflict of Interest: The authors have no conflicts of interest to declare.

\section{References}

1. Cribier A, Eltchaninoff H, Bash A, et al. Percutaneous transcatheter implantation of an aortic valve prosthesis for calcific aortic stenosis: first human case description. Circulation 2002;106:3006-8.

2. Webb JG, Chandavimol M, Thompson CR, et al. Percutaneous aortic valve implantation retrograde from the femoral artery. Circulation 2006;113:842-50.

3. Svensson LG, Tuzcu M, Kapadia S, et al. A comprehensive review of the PARTNER trial. J Thorac Cardiovasc Surg 2013;145:S11-6.

4. Adams DH, Popma JJ, Reardon MJ, et al. Transcatheter aortic-valve replacement with a self-expanding prosthesis. N Engl J Med 2014;370:1790-8.

5. Nishimura RA, Otto CM, Bonow RO, et al. 2014 AHA/ ACC guideline for the management of patients with valvular heart disease: executive summary: a report of the American College of Cardiology/American Heart Association Task Force on Practice Guidelines. J Am Coll Cardiol 2014;63:2438-88.

6. Latib A, Maisano F, Bertoldi L, et al. Transcatheter vs surgical aortic valve replacement in intermediate-surgicalrisk patients with aortic stenosis: a propensity scorematched case-control study. Am Heart J 2012;164:910-7.

7. Piazza N, Kalesan B, van Mieghem N, et al. A 3-center comparison of 1-year mortality outcomes between transcatheter aortic valve implantation and surgical aortic valve replacement on the basis of propensity score matching among intermediate-risk surgical patients. JACC Cardiovasc Interv 2013;6:443-51.

8. Thyregod HG, Steinbrüchel DA, Ihlemann N, et al. Transcatheter Versus Surgical Aortic Valve Replacement in Patients With Severe Aortic Valve Stenosis: 1-Year Results From the All-Comers NOTION Randomized Clinical Trial. J Am Coll Cardiol 2015;65:2184-94.

9. D'Errigo P, Barbanti M, Santini F, et al. Results of the 
OBSERVANT study: clinical characteristics and shortterm outcome of the enrolled population treated with transcatheter versus surgical aortic valve implantation. $G$ Ital Cardiol (Rome) 2014;15:177-84.

10. Tamburino C, Barbanti M, D'Errigo P, et al. 1-Year Outcomes After Transfemoral Transcatheter or Surgical Aortic Valve Replacement: Results From the Italian OBSERVANT Study. J Am Coll Cardiol 2015;66:804-12.

11. Schymik G, Heimeshoff M, Bramlage P, et al. A comparison of transcatheter aortic valve implantation and surgical aortic valve replacement in 1,141 patients with severe symptomatic aortic stenosis and less than high risk. Catheter Cardiovasc Interv 2015;86:738-44.

12. Muneretto C, Bisleri G, Moggi A, et al. Treating the patients in the 'grey-zone' with aortic valve disease: a comparison among conventional surgery, sutureless valves and transcatheter aortic valve replacement. Interact Cardiovasc Thorac Surg 2015;20:90-5.

13. Lange R, Bleiziffer S, Mazzitelli D, et al. Improvements in transcatheter aortic valve implantation outcomes in lower surgical risk patients: a glimpse into the future. J Am Coll Cardiol 2012;59:280-7.

14. Wenaweser P, Stortecky S, Schwander S, et al. Clinical outcomes of patients with estimated low or intermediate surgical risk undergoing transcatheter aortic valve implantation. Eur Heart J 2013;34:1894-905.

15. Kondur A, Briasoulis A, Palla M, et al. Meta-Analysis of Transcatheter Aortic Valve Replacement Versus Surgical Aortic Valve Replacement in Patients With Severe Aortic Valve Stenosis. Am J Cardiol 2016;117:252-7.

16. Hemmann K, Sirotina M, De Rosa S, et al. The STS score is the strongest predictor of long-term survival following transcatheter aortic valve implantation, whereas access route (transapical versus transfemoral) has no predictive value beyond the periprocedural phase. Interact Cardiovasc Thorac Surg 2013;17:359-64.

17. Piazza N, Wenaweser P, van Gameren M, et al. Relationship between the logistic EuroSCORE and the Society of Thoracic Surgeons Predicted Risk of Mortality score in patients implanted with the CoreValve ReValving system--a Bern-Rotterdam Study. Am Heart J 2010;159:323-9.

18. Thourani VH, Suri RM, Gunter RL, et al. Contemporary real-world outcomes of surgical aortic valve replacement in 141,905 low-risk, intermediate-risk, and high-risk patients. Ann Thorac Surg 2015;99:55-61.
19. Iturra SA, Suri RM, Greason KL, et al. Outcomes of surgical aortic valve replacement in moderate risk patients: implications for determination of equipoise in the transcatheter era. J Thorac Cardiovasc Surg 2014;147:127-32.

20. Kappetein AP, Head SJ, Généreux P, et al. Updated standardized endpoint definitions for transcatheter aortic valve implantation: the Valve Academic Research Consortium-2 consensus document. J Thorac Cardiovasc Surg 2013;145:6-23.

21. Thongprayoon C, Cheungpasitporn W, Srivali N, et al. AKI after Transcatheter or Surgical Aortic Valve Replacement. J Am Soc Nephrol 2015. [Epub ahead of print].

22. Généreux P, Kodali SK, Green P, et al. Incidence and effect of acute kidney injury after transcatheter aortic valve replacement using the new valve academic research consortium criteria. Am J Cardiol 2013;111:100-5.

23. Najjar M, Salna M, George I. Acute kidney injury after aortic valve replacement: incidence, risk factors and outcomes. Expert Rev Cardiovasc Ther 2015;13:301-16.

24. Thongprayoon C, Cheungpasitporn W, Srivali N, et al. Acute kidney injury after transcatheter aortic valve replacement: a systematic review and meta-analysis. Am J Nephrol 2015;41:372-82.

25. Smith $C$. Transcatheter vs. surgical aortic valve replacement in high risk patients with severe aortic stenosis: results from the PARTNER trial. Oral presentation presented at: American College of Cardiology Annual Scientific Sessions; April 3, 2011; New Orleans, USA.

26. Agarwal S, Parashar A, Kumbhani DJ, et al. Comparative meta-analysis of balloon-expandable and self-expandable valves for transcatheter aortic valve replacement. Int J Cardiol 2015;197:87-97.

27. Webb, J. One-year outcomes from the SAPIEN 3 trial. Oral Presentation presented at: Euro PCR 2015; May 7, 2015; Paris, France.

Cite this article as: Arora S, Misenheimer JA, Jones W, Bahekar A, Caughey M, Ramm CJ, Caranasos TG, Yeung M, Vavalle JP. Transcatheter versus surgical aortic valve replacement in intermediate risk patients: a meta-analysis. Cardiovasc Diagn Ther 2016;6(3):241-249. doi: 10.21037/ cdt.2016.03.04 